\title{
Microenvironment-mediated resistance to anticancer therapies
}

Cell Research (2013) 23:179-181. doi:10.1038/cr.2012.123; published online 4 September 2012

Resistance to molecularly targeted
therapies can result from genomic
alterations in the tumor cells that
reactivate oncogenic signaling. Less
is known of tumor cell-extrinsic
mechanisms of resistance to targeted
therapies. Two recent studies have
identified HGF as a soluble factor
capable of mediating resistance to
BRAF and HER2 inhibitors in a
paracrine manner. These new find-
ings suggest an important role for the
tumor microenvironment in mediat-
ing resistance to molecularly targeted
therapies.
Despite the continued development of novel therapeutics for the treatment of cancer, innate and acquired resistance remain intractable problems in clinical care. While the investigation of therapeutic resistance has not surprisingly centered on tumor cell-intrinsic mechanisms to date, recent findings have uncovered novel roles for the tumor microenvironment in modulating therapeutic efficacy. The importance of the tumor microenvironment in cancer initiation and progression is well established [1], but our understanding of the contributions of the microenvironment to therapeutic response, and innate and acquired resistance, is in its infancy.

The "tumor stroma" is often used as a catch-all term in referring to all nonmalignant cells, including endothelial cells, fibroblasts, and infiltrating leukocytes, as well as extracellular matrix proteins in the cancer microenvironment. Stromal signatures have been shown to have powerful prognostic value in predicting treatment outcome in breast cancer and lymphoma $[2,3]$ among others, and while these findings underscore the importance of the microenvironment in patient response there remain many questions as to the underlying mechanisms. Several studies have previously screened for tumor-stromal cell interactions capable of mediating chemo-resistance [4, 5], but the scale of two recently published high-throughput screens represents a significant increase in magnitude $[6,7]$ (Figure 1). For example, the group at the Broad Institute, screened the capacity of 23 stromal cell lines to alter the response of 45 cancer cell lines to 35 commonly used anticancer agents [6]. This study demonstrated that interactions between stromal and tumor cells have an extensive capacity to modulate response to therapy, particularly in the context of molecularly targeted agents.

The potential mechanisms by which the microenvironment can affect drug response and mediate resistance fall into the following four broad categories: (1) survival signals from soluble factors or cell adhesion molecules; (2) impaired drug delivery; (3) immunosuppression; (4) enhanced tumor regrowth. Cell adhesion to extracellular matrix proteins can suppress the response to chemotherapy through $\beta 1$ integrin-mediated survival signaling [8]. Similarly, soluble factors secreted by stromal cells, such as IL-6 and cathepsin proteases, have also been shown to blunt therapeutic efficacy [4, 5]. From a physiological perspective the microenvironment can contribute to intrinsic resistance by limiting delivery of chemotherapy into the tumor as reported for pancreatic ductal adenocarcinoma [9]. Targeting this stromal desmoplasia greatly enhances treatment response in preclinical models [9]. The immune system can also have an important role in treatment response, and an immunosuppressive microenvironment blunts response to cytotoxic therapy [10]. Finally, stromal cells are able to facilitate the rapid and efficient regrowth of the tumor, as observed following glioblastoma radiation treatment [11], and in this manner diminish the durability of treatment response.

While the role of the tumor microenvironment in mediating resistance to classical chemotherapy, radiation, and anti-angiogenic agents is thus emerging, virtually nothing is known about its effect on "molecularly targeted therapies". Over the past decade targeted therapies, which block activated oncogenic signaling pathways, have proven to be an effective therapeutic strategy with significant clinical success. Of particular note is the FDA approval of the small molecule BRAF inhibitor PLX4032/ vemurafenib for the treatment of BRAF mutant metastatic melanoma. Treatment in this patient population results in improved progression-free survival (PFS) and overall survival (OS), but the majority of patients develop resistance after 6-9 months [12]. Several tumor cellintrinsic mechanisms of BRAF inhibitor resistance have recently been identified, including BRAF splice variants in melanomas and feedback activation of EGFR signaling in colorectal carcinoma $[13,14]$. Little is known, however, about the potential role of the tumor microen- 
A

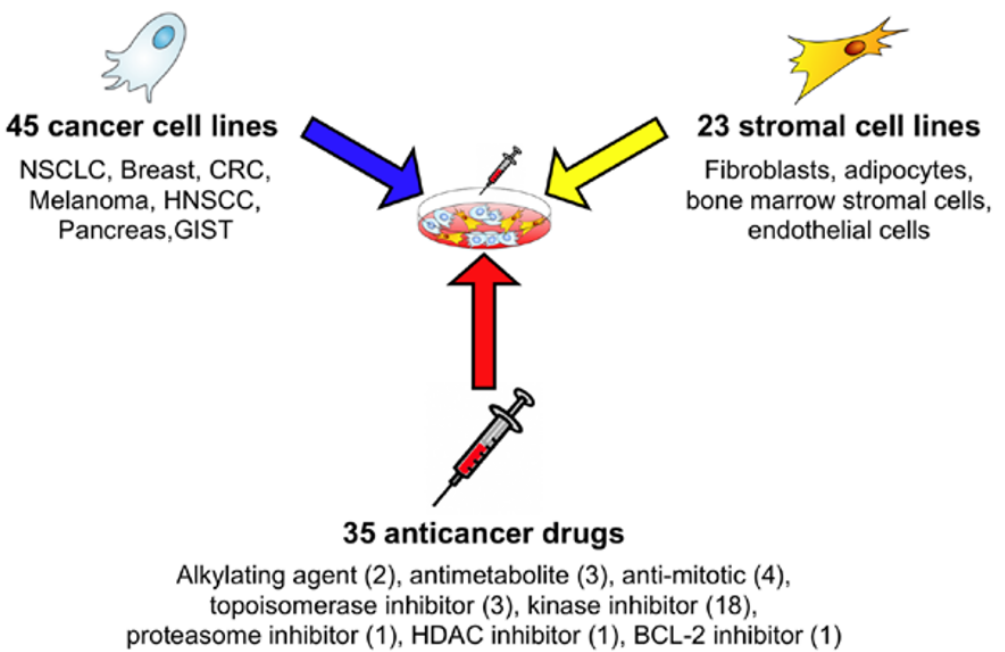

B

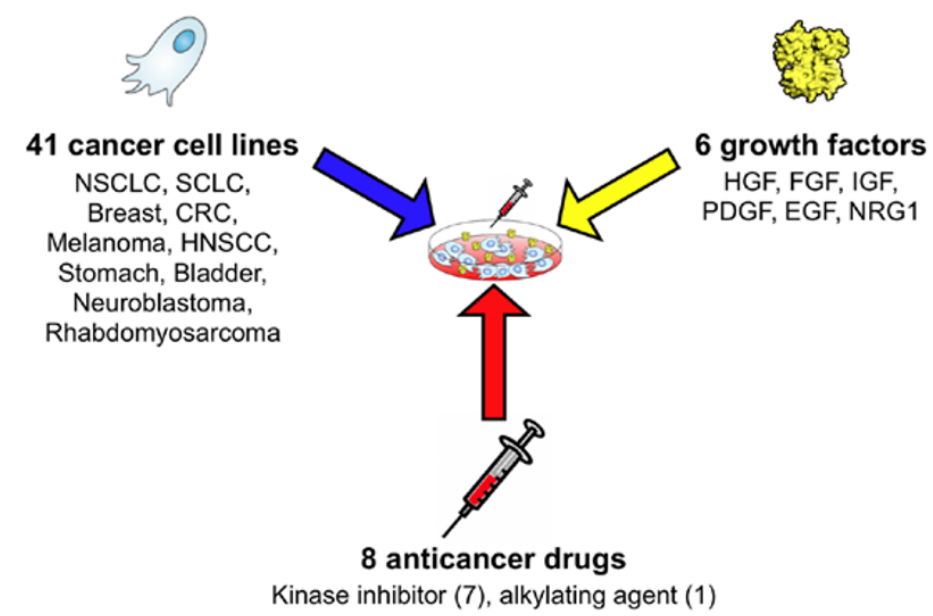

Figure 1 Overview of two high-throughput screens performed to identify stromal cells and RTK ligands capable of mediating chemoresistance. (A) Straussman and colleagues tested the ability of 23 stromal cell lines to protect 45 cancer cell lines against the activity of a panel of anticancer drugs with diverse mechanisms of action. Stromal cell-mediated resistance was observed for 16 out of the 35 drugs tested. (B) Wilson and colleagues tested the ability of growth factors to mediate resistance to molecularly targeted agents. Cancer cell lines were classified based on genotype including HER2, MET and FGFR amplification, EGFR and BRAF mutation, ALK translocation, and NRG1 and EGF-like ligand autocrine signaling. Each cell line was treated with the appropriate inhibitor indicated by their genotype. Five of the 6 RTK ligands tested demonstrated the ability to rescue cancer cells from molecularly targeted therapy. The individual cancer cell lines from different tumor types are indicated for each screen. Abbreviations: NSCLC, non-small cell lung cancer; CRC, colorectal cancer; HNSCC, head and neck squamous cell carcinoma; GIST, gastrointestinal stromal tumor; SCLC, small cell lung cancer; HDAC, histone deacetylase; RTK, receptor tyrosine kinase; HGF, hepatocyte growth factor; FGF, fibroblast growth factor; IGF, insulin-like growth factor; PDGF, platelet-derived growth factor; EGF, epidermal growth factor; NRG1, neuregulin 1.

vironment in BRAF inhibitor resistance and treatment failure.

Two recent articles add significantly to this body of work through the identification of hepatocyte growth factor (HGF) as a soluble factor capable of mediating resistance to BRAF inhibition in V600E BRAF mutant melanoma, as well as HER2 inhibition in HER2+ breast cancer [6, 7]. Hypothesizing that communication with stromal cells can contribute to chemoresistance, Straussman and colleagues performed a highthroughput screen to identify stromal cell interactions capable of mediating resistance to a broad panel of therapeutic agents with diverse mechanisms of action [6] (Figure 1A). Six fibroblast cell lines, of the 23 stromal cell lines tested, were able to completely rescue the viability of BRAF mutant melanoma cell lines treated with a BRAF inhibitor. The authors demonstrated that this protective activity was present in conditioned media from the fibroblast cell line, and thus not dependent on cell-cell contact. Through analysis of the fibroblast-conditioned media for 567 secreted factors, HGF was identified as the protective factor. This was confirmed by demonstrating that the resistance in question was sensitive to inhibition of MET, the cognate signaling receptor for HGF, using the small molecule crizotinib.

Wilson and colleagues at Genentech reached similar conclusions by prospectively screening the ability of several growth factors, including HGF, for the ability to protect oncogene-addicted cell lines from targeted kinase inhibition [7] (Figure 1B). Their results not only demonstrated the capability of HGF to attenuate BRAF inhibition in BRAF mutant melanoma cell lines, but also implicated receptor tyrosine kinase ligands as broadly relevant mediators of resistance to targeted therapy. They correlated the survival promoting functions of these growth factors with reactivation of the MAPK or PI3K/AKT pathways in a cell line-specific manner. This suggests that diversification of survival signaling input is critical to attenuating oncogene addiction and in this manner the tumor microenvironment has a potential role in the heterogeneity of RTK signal inputs. This is highly 
reminiscent of tumors refractory to anti-VEGF therapy where fibroblasts and infiltrating myeloid cells provide alternative angiogenic factors such as PDGF-C and Bv8 [15]. Whether this circumvention of oncogene addiction will occur in a restricted and targetable manner remains an open question.

It is possible that evaluation of the HGF/MET signaling axis in the tumor may indicate the susceptibility of that tumor to show resistance through this pathway. Indeed, higher expression of the MET receptor in different cancer cell lines correlated with their resistance to targeted therapy mediated by HGF [7]. Demonstrating the clinical importance of these findings, serum analysis of melanoma patients treated with BRAF inhibitors correlated high levels of circulating HGF with decreased PFS and OS [7]. Histological analyses of patient samples showed a negative correlation between stromal HGF expression and clinical response [6]. Unfortunately neither group identified the cellular origin of this "stromal" HGF expression in the context of metastatic melanoma. Straussman and colleagues infer fibroblasts as the cell type responsible for HGF expression given the HGF secretion by fibroblast cell lines and the stromal restriction of HGF expression in histological analysis of patient samples. Wilson and colleagues did not attempt to address the origin of the growth factors that they associate with innate resistance to targeted agents, and thus the actual cellular source of these secreted growth factors in the tumor microenvironment remains an important open question. Nonetheless, the patient analyses in both studies support the hypothesis of HGF-mediated resistance and suggest its potential as a prognostic biomarker.

Recently multiple groups have reported instances of changes in cell populations within the tumor microenvironment in response to chemotherapeutics, radiation and anti-angiogenic treatment. Increased populations of bone marrow-derived cells have been shown to aid tumor recovery and blunt cytotoxic therapeutic response and immunologic death [5, 10, 15-17]. It remains unclear to what extent treatment with targeted therapies might deplete or expand certain stromal populations, or alter their functional character. Intriguingly, the authors note that roughly half the patients that they analyzed showed increased HGF expression in matched tissue biopsies after treatment with vemurafenib [6], strongly suggesting that the microenvironment may be reactive to targeted therapy, and an active partner in acquired as well as intrinsic resistance.

The identification of stromal HGF as a mediator of vemurafenib resistance has important translational potential. Both groups suggest clinical investigation of concurrent treatment of BRAF inhibitors with the FDA-approved MET inhibitor crizotinib. Alternatively, trials designed to suppress tumor cellintrinsic or acquired resistance through combined BRAF/MEK inhibition may also be simultaneously suppressing microenvironmental-mediated resistance. Proponents of microenvironmental targeting, however, stress stromal cells as an ideal target and point of therapeutic intervention due to their inherent genomic stability.

These two high-throughput, yet relatively straightforward, screens have produced a wealth of data that has broadened our understanding of resistance to molecularly targeted therapies. The analyses presented in these two papers represent just a fraction of what is likely to be uncovered in the future from these expansive datasets. While it is clear that there is an important role for the tumor microenvironment, these studies have raised many intriguing questions. For example, it is unclear to what extent the tumor cells are dependent on the stroma, or whether they can effectively express these protective growth factors in an autocrine manner. Similarly, the heterogeneity of the stromal cellular response to molecularly targeted therapies within the tumor microenvironment remains undefined. Moreover, due to the absence of rigorous in vivo validation it remains uncertain from these studies $[6,7]$ what outcomes will result from additional targeting of supportive signaling from the microenvironment. Regardless, it is clear that the microenvironment has a significant impact on tumor cell signaling in the context of therapeutic intervention and thus represents an attractive therapeutic target.

\section{Oakley C Olson ${ }^{1,2}$, Johanna A Joyce ${ }^{1}$}

${ }^{1}$ Cancer Biology and Genetics Program, ${ }^{2}$ Louis V. Gerstner Jr. Graduate School of Biomedical Sciences, Memorial Sloan-Kettering Cancer Center, New York, NY 10065, USA

Correspondence: Johanna A Joyce E-mail: joycej@mskcc.org

\section{References}

1 Joyce JA, Pollard JW. Nat Rev Cancer 2009; 9:239-252.

2 Dave SS, Wright G, Tan B, et al. $N$ Engl J Med 2004; 351:2159-2169.

3 Finak G, Bertos N, Pepin F, et al. Nat Med 2008; 14:518-527.

4 McMillin DW, Delmore J, Weisberg E, et al. Nat Med 2010; 16:483-489.

5 Shree T, Olson OC, Elie BT, et al. Genes Dev 2011; 25:2465-2479.

6 Straussman R, Morikawa T, Shee K, et al. Nature 2012; 487:500-504.

7 Wilson TR, Fridlyand J, Yan Y, et al. Nature 2012; 487:505-509.

8 Sethi T, Rintoul RC, Moore SM, et al. Nat Med 1999; 5:662-668.

9 Olive KP, Jacobetz MA, Davidson CJ, et al. Science 2009; 324:1457-1461.

10 DeNardo DG, Brennan DJ, Rexhepaj E, et al. Cancer Discov 2011; 1:54-67.

11 Kioi M, Vogel H, Schultz G, et al. J Clin Invest 2010; 120:694-705.

12 Dummer R, Flaherty KT. Curr Opin Oncol 2012; 24:150-154.

13 Poulikakos PI, Persaud Y, Janakiraman M, et al. Nature 2011; 480:387-390.

14 Prahallad A, Sun C, Huang S, et al. Nature 2012; 483:100-103.

15 Crawford Y, Ferrara N. Trends Pharmacol Sci 2009; 30:624-630.

16 Shaked Y, Henke E, Roodhart JM, et al. Cancer Cell 2008; 14:263-273.

17 Acharyya S, Oskarsson T, Vanharanta S, et al. Cell 2012; 150:165-178. 